\title{
Epitaxial growth of aligned semiconductor nanowire metamaterials for photonic applications
}

\author{
By Otto L. Muskens, Silke L. Diedenhofen, Maarten H. M. van Weert, Magnus T. Borgström, Erik P. A. \\ M. Bakkers, and Jaime Gómez Rivas ${ }^{*}$
}

\begin{abstract}
We present a novel class of optical metamaterials consisting of high densities of aligned gallium phosphide (GaP) nanowires fabricated using metal-organic vapor phase-epitaxy. Starting from a gold island film as a catalyst for nanowire growth, a sequential combination of vapor-liquid-solid and lateral growth modes is employed to obtain a continuous tunability of the nanowire volume fraction from $7 \%$ to over $35 \%$. By choosing different crystallographic orientations of the GaP substrate, we design metamaterials with different nanowire orientations. The anisotropy of the nanowire building blocks results in strong optical birefringence. Polarization interferometry demonstrates a very large polarization extinction contrast of $4 \times 10^{3}$ combined with a sharp angular resonance which holds promise for optical sensing. Nanowire metamaterials may find applications in photonics, optoelectronics, non-linear and quantum optics, microfluidics, bio-, and gas sensing.
\end{abstract}

\section{Introduction}

Artificial materials exhibiting optical, electrical, or mechanical properties that are governed by the arrangement of their microscopic building blocks are of great importance in science and technology. Photonic metamaterials with subwavelength-scale structuring show effective optical properties markedly different from those of their individual constituent materials. ${ }^{[1]}$ One of these properties is optical formbirefringence, the difference in refractive index for different polarizations of light, resulting from the arrangement of anisotropic scatterers. ${ }^{[2]}$ Optical form-birefringence has been observed in various systems such as aligned carbon nanotube films, ${ }^{[3]}$ porous silicon, ${ }^{[4-6]}$ and liquid-crystal templated porous CdTe. ${ }^{[7]}$ In periodic structures like photonic crystals ${ }^{[8,9]}$ and gratings, ${ }^{[10]}$ large artificial birefringence occurs at certain frequencies near a resonance dictated by the periodicity. Subwavelength periodic structures in the visible are usually hard to produce and involve complex nanofabrication techniques with tight design tolerances. Novel methods to fabricate photonic materials reproducibly over large areas are of great importance in applications such as liquid crystal display technology, ${ }^{[11}$ integrated photonics, ${ }^{[12]}$ and optical sensing. ${ }^{[5,14,15]}$ Artificial metamaterials consisting of porous material at low volume fraction have recently been employed in broadband antireflection coatings. ${ }^{[15]}$

Here, we report on the bottom-up fabrication of a novel class of photonic metamaterials based on aligned semiconductor nanowires, exhibiting large artificial birefringence and optical polarization contrast. Semiconductor nanowires are at the focus of a rapidly expanding research field because of their promise as a building block for devices and applications. ${ }^{[16]}$ For single semiconductor nanowires, large anisotropy of the absorption and emission of light has been demonstrated by polarization-dependent luminescence measurements. ${ }^{[17]}$ This anisotropy was explained by the different polarizabilities of a thin cylinder for polarizations perpendicular and parallel to its axis. For ensembles of aligned nanowires, polarization anisotropy results in large optical birefringence. ${ }^{[18]}$ Semiconductor nanowires can be grown heteroepitaxially over large areas on most crystalline substrates including silicon ${ }^{[19,20]}$ and InP using the versatile bottom-up process of metal-organic vapor phaseepitaxy (MOVPE). Excellent spatial control over local growth allows straightforward integration with waveguides and photonic nanostructures. ${ }^{[21]}$ The nanowire morphology is complementary to inverted network materials such as porous silicon, which have found important applications in chemistry and biology. ${ }^{[22]}$ This open morphology of a nanowire ensemble compared to nanometer-sized pores in inverted networks will be superior for applications dealing with large molecules and liquids, for example in microfluidic environments. ${ }^{[23]}$ Integration of III-V nanowires with silicon ${ }^{[20]}$ will lead to hybrid nanowire metamaterials that will benefit from existing functionalization methods ${ }^{[22]}$ while maintaining the electrical and optical properties of the III-V backbone. High-density nanowire metamaterials will also be of importance for nextgeneration photovoltaic designs. ${ }^{[24,25]}$

\section{Results and discussion}

\subsection{Nanowire growth}

High-density ensembles of gallium phosphide ( $\mathrm{GaP}$ ) nanowires were grown in a MOVPE reactor following the procedure outlined in Section 4. The substrate is a single-crystalline GaP wafer covered with a $3-\AA$ thin gold film. The gold film provides a homogeneous coverage of the whole surface with small, uniformously distributed islands. ${ }^{[26]}$ Catalytic growth

$\left[{ }^{\star}\right] \quad$ Dr. O. L. Muskens, S. Diedenhofen, M. H. M. van Weert, Dr. J. Gómez Rivas FOM Institute for Atomic and Molecular Physics AMOLF c/o Philips Research Laboratories, High Tech Campus 4, 5656 $A E$, Eindhoven, The Netherlands, E-mail: rivas@amolf.nl M. H. M. van Weert, current address: Kavli Institute of Nanoscience, Delft University of Technology, Lorentzweg 1 , 2628CJ, Delft, The Netherlands

Dr. M. T. Borgström, Dr. E. P. A. M. Bakkers

Philips Research Laboratories, High Tech Campus 4, 5656 AE, Eindhoven, The Netherlands

Dr. M. T. Borgström, current address: Nanometer Structure Consortium, Lund University, P.O. Box 118, S-221 00 Lund, Sweden

[**] We thank G. Immink, W. van der Einden, and E. Evens for their technical assistance, F. Holthuysen and P. Breijmer for SEM analysis, and M. Verheijen for TEM analysis. This work is part of the research program of the the "Stichting voor Fundamenteel Onderzoek der Materie (FOM)", which is financially supported by the "Nederlandse Organisatie voor Wetenschappelijk Onderzoek (NWO)", and is part of an industrial partnership proaram between Philips and FOM. 
using the vapor-liquid-solid (VLS) process ${ }^{[27-29]}$ results in nanowires with a diameter determined by the size of the catalyst particles. The length of nanowires is set via the VLS growth time. Subsequently, the photonic strength of the material is tuned via the diameter of the wires by switching from the vapor-liquid-solid to the lateral growth mode. In this step, the nanowire layer is gradually filled up with semiconductor material at a higher temperature $\left(630^{\circ} \mathrm{C}\right)$ of the MOVPE chamber where the kinetic hindrance is overcome. The lateral growth process is limited by diffusion of precursor gases into the dense nanowire layer, which in practice limits this approach to nanowires shorter than $2 \mu \mathrm{m}$. Table 1 gives an overview of the nanowire materials of this study, grown on various substrate orientations using a combination of VLS and lateral growth.

\subsection{Structural characterization}

The morphology of the nanowire materials was investigated using both top-view and cross-sectional scanning electron microscopy (SEM). Figure 1(a) shows a top-view SEM image of sample A, after a very short period of VLS growth. The image reveals a density of nanowire growth centers of around 390 sites per $\mu \mathrm{m}^{2}$. The histogram of diameters (bottom graph of Fig 1a) shows a lognormal distribution around an average of $18.7 \mathrm{~nm}$. Since sites smaller than $\sim 10 \mathrm{~nm}$ cannot be discerned in the SEM image, the measured distribution is slightly biased toward larger diameters. The volume fraction of growth centers per unit area is estimated as $12 \pm 2 \%$ on the basis of SEM contrast analysis.

Gallium phosphide nanowires of several micrometers in length were grown by increasing the VLS growth time to $36 \mathrm{~min}$. Figure 1(b) shows a cross-sectional SEM image of the nanowire layer grown on a (111)B GaP substrate. The nanowires are aligned perpendicular to the substrate due to the preferential growth in the $<111>B$ direction. The thin wires show some bending toward the top, probably caused by an enhanced flexibility of the semiconductor material at these very small diameters. The distribution of nanowire diameters obtained from the SEM image is presented in the bottom graph of Fig. 1(b), showing a distribution centered around $27 \mathrm{~nm}$ diameter, with a polydispersity given by the standard deviation of $\pm 20 \%$. The nanowire size distribution is comparable to that of the nucleation sites in Fig. 1(a), indicating that the nanowire diameter is determined by the metal catalyst particle. In Fig. 1(b) as well as in top-view SEM images (not shown), the nanowire volume fraction appears lower than the initial density of growth centers which we assign to competitive growth and spontaneous growth interruption of wires. From the number density and diameters of full-grown wires we estimate a volume fraction of $8 \pm 4 \%$ as shown in Table 1. This value is corroborated by gravimetric analysis of the sample before and after growth.

The preferential growth of GaP nanowires in the $<111>\mathrm{B}$ direction was used to obtain nanowire ensembles with nonvertical orientations by changing the crystallographic orientation of the substrate. Figure 2(a) shows cross-sectional SEM images of GaP nanowires grown from a $\mathrm{GaP}$ (100) substrate, for a section cleaved along the (110)-plane. This cleaving orientation provides a projection perpendicular to the growth plane of the $<111>$ B nanowires, i.e. the wires are oriented in the plane of the SEM image. We observe a high-density distribution of nanowires with a large fraction of the wires under an angle of around $35^{\circ}$ with respect to the surface of the substrate. Some wires are observed that are oriented in the $<100>$ direction, and some in higher order $<11 \mathrm{x}>\mathrm{B}$ directions $(\mathrm{x}>1)$. The preferential growth will be exploited in Sections 2.4 and 2.5 to control the direction of the optical axis, and thus the birefringence, in the nanowire material. The average wire diameter for these materials was determined from SEM images and is shown in Table 1. From these diameters we have estimated the increase in volume fraction of the layers upon lateral growth, as indicated in Table 1 .

The crystalline properties of nanowires fabricated using VLS and lateral growth were analyzed using transmission electron microscopy (TEM) and energy dispersive X-ray (EDX) analysis. Figure 3 shows TEM micrographs of wires taken from the (100) GaP metamaterials $\mathrm{C}(\mathrm{a}, \mathrm{b})$ and $\mathrm{F}(\mathrm{c}, \mathrm{d})$ of Table 1 . The wires from material $\mathrm{C}$ have diameters ranging from approximately 10 to $20 \mathrm{~nm}$, no tapering was observed. The wires from material $\mathrm{F}$, with additional lateral growth, are considerably thicker: diameters exceeding $100 \mathrm{~nm}$ are observed. The diameter of the original gold particle (see inset) gives a good indication of thickness prior to sidewall growth. The contrast lines, orthogonal to the length direction of the wires, observed for both the thin and the thick wire, correspond to twin domains with alternating orientations along the $<111>$ axis of the wire. Remarkably, the upper segment of the wire in Fig. 3(c) is free of twin boundaries. The presence of a single-crystalline end-segment was observed only for nanowires from sample F, indicating that this $100-\mathrm{nm}$ long segment results from competing VLS growth during the lateral growth step. The nanowires have the zincblende structure, as determined from fast-fourier-transforms of HRTEM images such as that in Fig. 3(d). EDX studies of an area containing a group of nanowires (without any gold particles included) revealed $\mathrm{Ga}$ and $\mathrm{P}$ with equal stoichiometry. A more detailed TEM analysis of GaP nanowire growth using the same MOVPEsystem was presented in Ref. 29.

\subsection{Optical characterization}

An important application of porous form-birefringent materials is as an optical sensor using polarization interferometry. ${ }^{[4-6]}$ The resolution of such a device depends strongly on the strength of the birefringence and on the contrast between the orthogonal polarization states. The goal of our research is to fabricate a metamaterial that combines a very large optical anisotropy in the optical frequency domain with a high degree of polarization contrast. In order to achieve this combination we have chosen to use gallium phosphide $(\mathrm{GaP})$ nanowires. Gallium phosphide combines the largest refractive index in the visible of around 3.4 with very low absorption below its indirect electronic bandgap located at $2.26 \mathrm{eV}$ (548 $\mathrm{nm}){ }^{[30]}$ The polarizability of nanowires strongly depends on their orientation with respect to the optical field. As a consequence, nanowires show a large anisotropy in their optical response. ${ }^{[17,18,31]}$ Here, we investigate the optical properties of nanowire layers as a function of their density and alignment.

An ideal optical metamaterial consists of scattering building blocks that are much smaller than the optical wavelength in the medium. Under this condition the arrangement of scatterers results in a material with a refractive index determined by effective medium theory. To test this assumption for the nanowire metamaterials under study, we have performed white-light transmission spectroscopy using the procedure described in Section 4. Figure 4(a) shows the transmission spectra for samples C-F together with a bare (100) GaP substrate (black line). For all samples, a sharp absorption edge is present due to the electronic bandgap of $\mathrm{GaP}$ at $548 \mathrm{~nm}$. The reference sample shows an overall flat transmission below $100 \%$ due to Fresnel reflection losses, with a slight interference contribution from a thin-film $\mathrm{SiO}_{2}$ protective coating at the backside of the substrate. For the samples C-F with increasing lateral growth times, a strongly wavelength dependent transmission is observed, which can be assigned to Rayleigh scattering of the thin nanowires (dash-dotted lines), as shown below. The scattering losses increase considerably for thicker nanowires and toward shorter wavelengths. Above $900 \mathrm{~nm}$, the optical transmission is only slightly reduced with respect to the bare substrate.

We have interpreted the strong wavelength dependence of the transmission through the nanowire material using a simple Beer's law for the coherent beam assuming Rayleigh scatterers, given by

$$
T=T_{0} \exp \left(-L / l_{e}\right)
$$

where $T_{0}$ denotes the Fresnel transmission coefficient through the layer, $l_{e}(\lambda)=A \lambda^{4}$ is the wavelength dependent scattering length containing the typical fourth power dependence on wavelength (Rayleigh scattering), and $L$ denotes the sample thickness. Fits to the data are indicated by the dashdotted lines in Fig. 4(a), which show good agreement for wavelengths above $700 \mathrm{~nm}$. For shorter wavelengths deviations arise because of the breakdown of the Rayleigh scattering approximation for finite size scatterers. Figure 4(b) represents the extinction mean free path at the wavelength of the experiments below, $\lambda=690 \mathrm{~nm}$. For a small cylindrical particle in the long-wavelength limit, the scattering mean free path is expected to vary as the inverse fourth power of the diameter. ${ }^{[32]}$ This dependence is shown as a line in the inset Fig. 4(b) and gives good 
agreement for the thin nanowire materials with average wire diameters below $<\mathrm{d}>=50 \mathrm{~nm}$. For the thicker nanowires, finite size effects result in a more complex dependence of the scattering cross-section on particle diameter and wavelength. ${ }^{[32]}$

\subsection{Angle-dependent polarization interferometry of nanowires on a (111)B GaP substrate}

For a material consisting of vertically aligned semiconductor nanowires, the polarization directions perpendicular to the wire axis have a lower refractive index than the direction parallel to the nanowires. ${ }^{[2]}$ An optical beam traveling through this material will therefore have a different velocity depending on its polarization. We used the method of polarization interferometry as explained in Section 4 to investigate the birefringence in the nanowire materials. Due to the preferential orientation of the wires on the (111)B substrate, the optical birefringence is expected to vary strongly between normal and in-plane incidence of light. The ratio of intensities in the cross-polarized $I_{\perp}$ and parallel polarized $I_{\|}$transmission channels is a measure for the degree of rotation of the polarization vector. This method, closely related to the more general technique of ellipsometry, has been widely used for the determination of optical birefringence as well as in optical sensors. ${ }^{[4-6]}$ Figure 5(a) shows this intensity contrast $I_{\perp} / I_{\|}$over more than 4 orders of magnitude for the nanowire material $B$. For comparison we have also plotted the result for a bare $\mathrm{GaP}$ (111)B substrate. Bulk GaP itself has only very small intrinsical birefringence $\left(<10^{-5}\right){ }^{[33]}$ which gives a neglegible contribution to the retardation. The small change in the ratio for the bare substrate can be completely explained by the difference in Fresnel transmission coefficients for the in-plane $(p-)$ and out-of-plane $(s-)$ polarizations, leading to a distortion of the initial polarization state. The nanowire material shows a much stronger dependence on angle, with a maximum occurring at $72^{\circ}$. This maximum corresponds to half-wavelength retardation, where the polarization vector has been converted entirely to the cross-polarized state. The amplitude of this maximum in $I_{\perp} / I_{\|}$is determined by the suppression of the original parallel state $I_{\|}$. By correcting the input state for polarization-dependent reflection losses, we have been able to further improve the maximum contrast by two orders of magnitude, as shown by the thin line in Fig. 5(a) This increase of the amplitude is associated with a narrowing of the fullwidth-at-half-maximum of the maximum from $9^{\circ}$ to $1.1^{\circ}$.

The intensity ratio $I_{\perp} / I_{\|}$for the reflected light is shown in Fig. 5(b). The effect of nanowire birefringence on the polarization state is here two times larger than in transmission since the optical beam passes through the nanowire layer twice. As a consequence, the location of the maximum in the intensity ratio occurs at an angle of incidence of $54^{\circ}$. Again a sharp maximum in the contrast is obtained after correction of the input state, with an extinction of the orthogonal polarization exceeding $4 \times 10^{3}$. This value exceeds the measured ratio in transmission, which we attribute to a reduced scattering background at smaller angles of incidence. Both the stronger angle-dependence of the intensity ratio and the higher extinction result in a reduced full-width-at-half-maximum of $0.45^{\circ}$. At angles above $60^{\circ}$, a complex oscillation pattern arises that can be assigned to thin-film interference fringes in the nanowire layer. These fringes distort the effects of retardation at large angles.

Accurate estimates of the material refractive indices for the (111)B GaP nanowires in Fig. 5 were obtained by fitting the angle-dependent transmission using a transfer matrix model accounting for Fresnel reflections at the various interfaces and for the uniaxial birefringence of the nanowire layer. The results of these fits are represented by the thin black lines in Fig. 5 and show good quantitative agreement with the experimental results. The resulting birefringence coefficient $\Delta n$ was determined as $0.047 \pm 0.005$, where the error bar results from the uncertainty in the optical thickness of the birefringent layer.

To investigate the use of angle-dependent nanowire birefringence for optical sensing we calculated the change of the contrast function for a small change of the birefringence parameter. The result is given in Fig. $6(\mathrm{a}, \mathrm{b})$. Again the reflectivity contrast of Fig. 5(b) is plotted around the maximum, but now with a fit including the correction for the input polarization (dashed line, black). Figure 6(b) compares this fit to a calculation using the same parameter except for a small change in birefringence $\Delta n$ of $10^{-3}$. We find a displacement of the contrast maximum over an angle of $0.5^{\circ}$, i.e. one linewidth of the inteferometric peak structure We estimate that much smaller changes, i.e. below $10^{-4}$, will be measurable using sensitive differential techniques on the slope of the peak. This corresponds well to sensitivities reported for porous silicon, in principle allowing for sensing of molecules in the ppm range. ${ }^{[6]}$

\subsection{In-plane birefringence of nanowires on a (100) GaP substrate}

To demonstrate the versatility of the bottom-up fabrication of nanowire photonic materials, we controlled the direction of the birefringence using the special crystallographic growth directions of semiconductor nanowires. Conclusive evidence of this preferential nanowire orientation is given by the optical birefringence experiment presented in Fig. 7 and explained in Section 4. Here the transmission of a normally incident wave on the sample is measured while rotating the nanowire sample azimuthally, i.e., around the normal of the sample surface. We observe a typical fourfold pattern that is indicative of in-plane birefringence as can be understood using simple arguments. At $-45^{\circ}$ and $45^{\circ}$ azimuthal angle the nanowires are aligned respectively parallel and perpendicular to the incident polarization vector, therefore no retardation takes place. This results in a zero of the ratio $I_{\perp} / I_{\|}$. At $0^{\circ}$ and $90^{\circ}$ azimuthal angle, the fast optical axis of the nanowires is at $45^{\circ}$ with the input polarization, giving rise to retardation and hence to a maximum in the ratio $I_{\perp} / I_{\|}$. The amount of retardation is relatively small for thin wires (sample $\mathrm{C}$ ) but increases strongly with increasing nanowire filling fraction (sample F). For sample $\mathrm{F}$ a maximum ratio $I_{\perp} / I_{\|}$larger than unity is obtained, indicating that we have fabricated an in-plane quarter-wavelength retarder using semiconductor nanowires (c.f. Section 4).

From the retardation, denoted here as $\delta$, we can derive the in-plane birefringence using the definition ${ }^{2,5}$

$\delta=\Delta n L$,

where $L$ denotes the thickness of the nanowire layer and $\lambda$ is the wavelength in vacuum. The retardance can be obtained from the contrast ratio via the relation

$$
\left(I_{\perp} / I_{/ /}\right)_{\max }=\tan \pi \delta / \lambda
$$

resulting in the values of the birefringence parameter $\Delta \mathrm{n}$ presented in Table 1. The error in the obtained birefringence values is mainly determined by an uncertainty in the layer thickness resulting from the various growth directions of nanowires on (100) GaP, as observed in Fig. $2(a, b)$.

From the maximum ratio of $I_{\perp} / I_{||}=1.5$ for the (100) GaP nanowire material $\mathrm{F}$ in Fig. 7 , we calculate a retardation over $\lambda / 4$, hence the nanowires act as a quarter waveplate retarder at an optical wavelength of $690 \mathrm{~nm}$. Between the materials $\mathrm{C}-\mathrm{F}$ before and after additional lateral growth, we find a variation over 2 orders of magnitude in the birefringence value $\Delta \mathrm{n}$. The lateral growth step results in a significant increase of the nanowire volume fraction by more than a factor of five. From effective medium theories such as Maxwell-Garnett ${ }^{[18]}$ or Bruggeman, ${ }^{[6]}$ we may expect a concomitant increase of the optical anisotropy in the low-volume fraction regime. For the thin-wire samples $\mathrm{C}$ and $\mathrm{D}$, there is still an additional reduction of birefringence by almost an order of magnitude that is not accounted for by effective medium models. We expect that this reduction is due to orientational disorder in the layer which is more pronounced for thin wires due to their abovementioned flexibility. ${ }^{[18]}$ Note that in these calculations we did not include effects from scattering, since to first order these are not expected to affect the polarization properties of the coherent beam. In the analysis, the specific Y-shaped morphology of the nanowires on (100) $\mathrm{GaP}$ is not taken into account since we only consider light incident perpendicular to the surface. For the optical response at oblique angles of incidence, a more detailed analysis, including the full, threedimensional nanowire orientations, will be required, which goes beyond the current investigation. 


\section{Conclusions}

In conclusion we have demonstrated that bottom-up fabrication of epitaxially-grown, semiconductor nanowires using MOVPE yields highly anisotropic photonic metamaterials. We have demonstrated control over both the morphology and the alignment of the nanowire metamaterials using a combination of VLS- and lateral growth and by growing on substrates with different crystallographic orientations. Using white ligh transmission measurements, we have observed a strongly wavelengthdependent optical extinction, following Rayleigh scattering at long wavelengths and for small nanowire diameters. For nanowire diameters above $50 \mathrm{~nm}$ a transition is found to the non-Rayleigh scattering regime. Although the short scattering mean free paths may pose limitations for application as optical elements, it may also be advantageous for trapping light inside structures such as solar cells and photodetectors. The exploration presented here may therefore be relevant for future designs and concepts in those areas.

In the polarization interferometry experiment, vertically aligned nanowire layers show very sharp features with a high dynamic range both in angular-dependent transmission and in reflection. In the latter configuration the effect of birefringence is twice as large due to the double passage through the layer. However, this configuration also suffers more strongly from spurious reflections and thin-film Fabry-Pérot fringes. The very high dynamic range of $10^{6}$ combined with high angular resolution make polarization interferometry an ideal tool for optical sensing. The sharp features obtained for long and thin nanowire samples are highly promising and at least comparable to the porous silicon materials used in earlier work. ${ }^{[5,6,13]}$ The fact that $\mathrm{GaP}$ phosphide has a relatively high-energy bandgap therefore allows a significant extension into the visible spectrum. The MOVPE method allows us to reproduce these nanowire layers in a controlled way, yielding constant optical birefringe values within the experimental error of $10 \%$ between runs.

Although we focused our investigation on gallium phosphide nanowires, nanowire metamaterials can be grown using any group III-V, II-VI, or IV semiconductor on any crystalline surface, offering the possibility of visible, near- and mid-infrared birefringence. Advantages of the bottom-up fabrication of the photonic nanowire birefringent materials are the high degree of control over local growth, the possibility of large area growth without the need of complex nanofabrication techniques, and the compatibility of the growth with silicon technology. nanowires were obtained from the layer by wiping of the sample over a copper grid. Statistical analysis of SEM images was done using ImagePro software. The diameter distribution of nucleation sites was obtained from contrast-analysis of the SEM image, yielding the area per nucleation site. Effective diameters were obtained from these areas using a cylindrical approximation, while the error was estimated from the uncertainty in the contrast threshold. The distribution of nanowire diameters was obtained by measuring, by hand, the diameters of 100 individual nanowires in the SEM images using the ImagePro software.

Optical characterization: Optical transmission spectra were measured using a Yokogawa AQ4303 fiber-coupled white light source. The fiber output was collimated using an achromatic objective and impinged perpendicular to the nanowire layer. Broadband polarization filters were used to select the incident and detected polarization states. The spectrum of transmitted light was measured using an Ocean Optics USB2000 spectrometer. A dynamic range of $10^{4}$ was obtained by combining two spectra with acquisition times of 0.1 and $2 \mathrm{~s}$. All transmission spectra were normalized to the spectrum of the white-light incident beam.

Angle-dependent polarization interferometry: The experimental scheme followed the general procedure presented in Refs. 4-6 and consisted of an angle-dependent reflection and transmission setup with a resolution of $0.1^{\circ}$ and polarization sensitivity of $1: 10^{5}$. A $632.8 \mathrm{~nm} \mathrm{He:Ne} \mathrm{laser} \mathrm{and} \mathrm{a} 690$ $\mathrm{nm}$ diode laser were used for the experiments. The polarization of the input beam was set to $45^{\circ}$ with respect to the plane of incidence. The rotation of the polarization vector after propagation through the nanowire layer was measured via the parallel- $\left(I_{\| \mid}\right.$.) and cross-polarized $\left(I_{\perp}\right)$ intensities (relative to the input polarization). The angle of incidence of the light was varied between 0 and $90^{\circ}$. The rotation of the polarization vector due to retardation inside the nanowire layer is measured via the polarization ratio $I_{\perp} / I_{\|}$. A pole in this ratio indicates a complete conversion of the original polarization state to a linear polarized orthogonal state, i.e. a retardation of $\lambda / 2$. Retardation of $\lambda / 4$ results in circular polarized output light, hence an equal contribution of the original and orthogonal state, which is measured as an $I_{\perp} / I_{\|}$ratio of 1 . For the (100) GaP materials, the polarization ratio was measured as a function of the azimuthal orientation of the nanowire layer.

Table 1. List of samples, with VLS and lateral growth times, nanowire diameter (standard deviation), length, and birefringence $\Delta \mathrm{n}$.

\section{Experimental}

MOVPE fabrication of nanowire layers: A gallium phosphide $(\mathrm{GaP})$ substrate was used with both sides mechanically and chemically polished to optical quality. A $500-\mathrm{nm} \mathrm{SiO}_{2}$ was deposited on the back surface using plasma deposition to prevent rough etching of this side. The substrate was etched in $\mathrm{HNO}_{3}: \mathrm{HCl}: \mathrm{H}_{2} \mathrm{O}(2: 3: 3)$ at $80{ }^{\circ} \mathrm{C}$ for $2 \mathrm{~min}$. Immediately after this step, a gold film with a thickness of $3 \AA$ was deposited on the front surface, which was either a P-terminated (111)B or a (100) interface. Subsequently the

\begin{tabular}{llllllll}
\hline No. & $\begin{array}{l}\text { GaP } \\
\text { substrate } \\
\text { orientation }\end{array}$ & $\begin{array}{l}\text { VLS } \\
\text { growth } \\
(\min .)\end{array}$ & $\begin{array}{l}\text { Lateral } \\
\text { growth } \\
(\mathrm{min})\end{array}$ & $\begin{array}{l}\text { Average } \\
\text { diameter } \\
<\mathrm{d}>(\mathrm{nm})\end{array}$ & $\begin{array}{l}\text { Length } \\
(\mu \mathrm{m})\end{array}$ & $\begin{array}{l}\text { Volume } \\
\text { fraction } \\
(\%)\end{array}$ & $\begin{array}{l}\text { Birefrin- } \\
\text { gence } \\
\Delta \mathrm{n}[\mathrm{a}]\end{array}$ \\
\hline A & $(111) \mathrm{B}$ & 2 & 0 & $19 \pm 6$ & $0.3 \pm 0.1$ & -- & -- \\
B & $(111) \mathrm{B}$ & 36 & 0 & $27 \pm 6$ & $4.6 \pm 0.2$ & $8 \pm 4$ & 0.047 \\
C & $(100)$ & 17 & 0 & $22 \pm 4$ & $1.2 \pm 0.1$ & $7 \pm 4$ & 0.003 \\
D & $(100)$ & 17 & $3 \min 20 \mathrm{~s}$ & $31 \pm 3$ & $1.3 \pm 0.1$ & $15 \pm 5$ & 0.011 \\
E & $(100)$ & 17 & $8 \min 20 \mathrm{~s}$ & $43 \pm 10$ & $1.4 \pm 0.2$ & $25 \pm 5$ & 0.099 \\
F & $(100)$ & 17 & $13 \min 20 \mathrm{~s}$ & $72 \pm 19$ & $1.6 \pm 0.2$ & $35 \pm 5$ & 0.209 \\
\hline
\end{tabular}
samples were transferred to a low-pressure (50 mbar) MOVPE system (Aixtron 200). Epitaxially oriented nanowires were grown in the VLS growth mode at an elevated temperature of $420{ }^{\circ} \mathrm{C}$ using trimethylgallium (TMG) and phosphine $\left(\mathrm{PH}_{3}\right)$ with molar fractions of $\chi_{\mathrm{TMG}}=9.1 \times 10^{-5}$ and $\chi_{\mathrm{PH} 3}=15.0 \times 10^{-3}$ respectively, as precursors in a total flow of $6.01 / \mathrm{min}$ using hydrogen $\left(\mathrm{H}_{2}\right)$ as carrier gas. Radial growth on the nanowire sidewalls was carried by switching off TMG during a temperature ramp to $630{ }^{\circ} \mathrm{C}$ after which the TMG flow was reintroduced into the reactor. After growth, the samples were cooled down in $\mathrm{PH}_{3}$ containing atmosphere.

Characterization: SEM images were made using a Nova NanoSEM 600 microscope by FEI Company. TEM, HRTEM, and EDS studies were performed using a Tecnai F30ST microscope at $300 \mathrm{kV}$. Individual

[a] Systematic error in birefringence values is $\pm 10 \%$ of $\Delta \mathrm{n}$ 

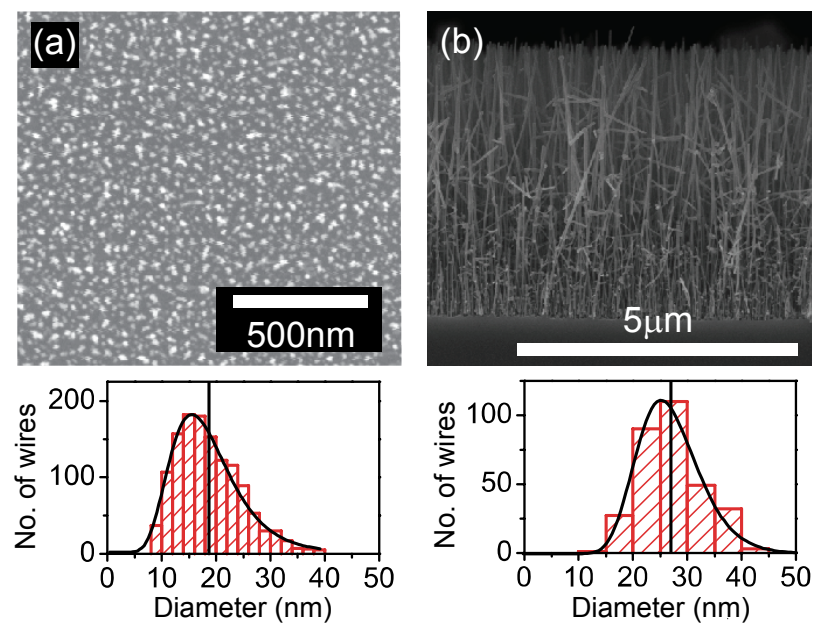

Figure 1. (a) Scanning electron micrograph (top view) of nanowire nucleation sites on sample A. (b) Cross-sectional SEM micrograph of a cleaved section of sample B. Bottom graphs of $(a, b)$ show size histograms of the nucleation sites and nanowires, respectively, with lognormal fits (black lines).
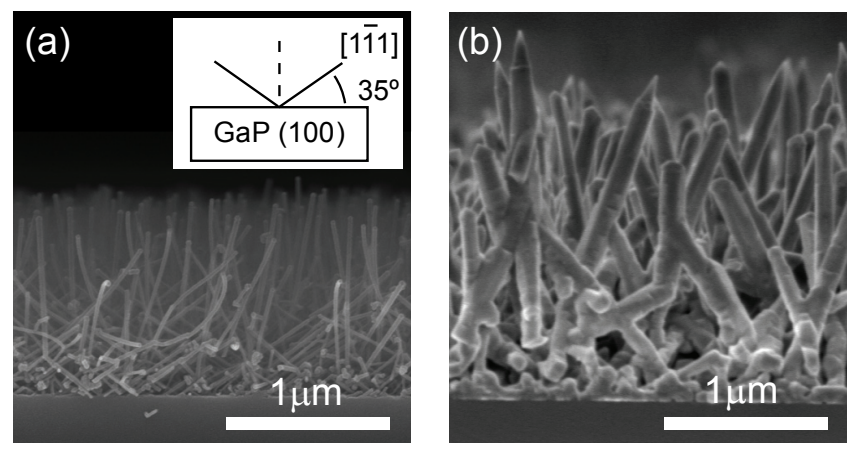

Figure 2. (a) Cross-sectional SEM image of a cleaved (110)-section of nanowire metamaterial $\mathrm{C}$, with (inset) schematic overview of crystallographic growth direction of the nanowires on the (100) GaP substrate. (b) Same for material F.
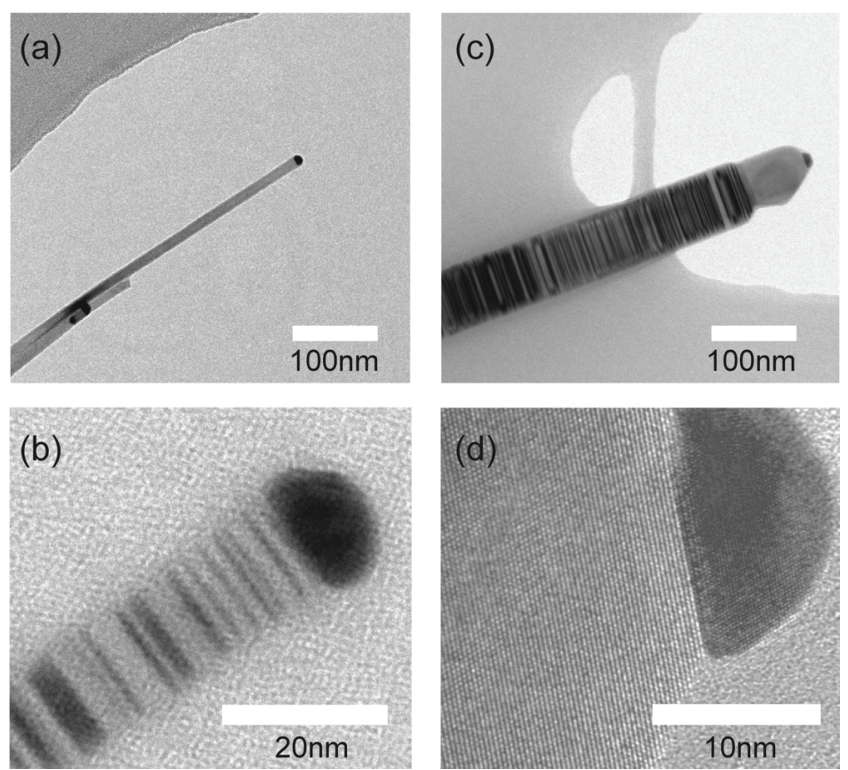

Figure 3. Brightfield transmission electron microscopy (TEM) image of $\mathrm{GaP}$ nanowires originating from $(\mathrm{a}, \mathrm{b})$ layer $\mathrm{C}$ and $(\mathrm{c}, \mathrm{d})$ layer $\mathrm{F}$. $(b, d)$ HRTEM images of nanowire tips showing crystalline structure. The small dark region at the tip of each nanowire indicates the gold catalyst particle which remains located on top of the wire during growth.

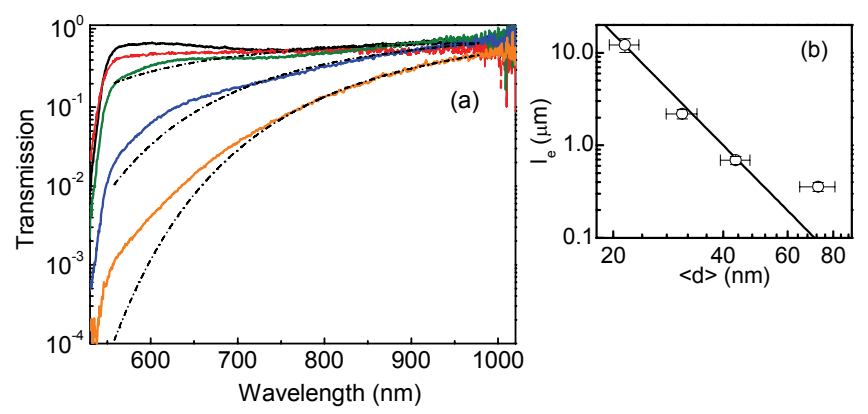

Figure 4. (a) Transmission spectrum of a white light beam through a GaP substrate (black line) and (100) GaP nanowire metamaterials $C$ - $F$ (red, green, blue, orange lines) with increasing nanowire diameters. Dash-dotted lines are fits using Rayleigh scattering model. (b) Extinction mean free path $\mathrm{I}_{\mathrm{e}}$ at $690 \mathrm{~nm}$ wavelength for materials $\mathrm{C}-\mathrm{F}$, (line) fit proportional to $<\mathrm{d}>^{-4}$. 


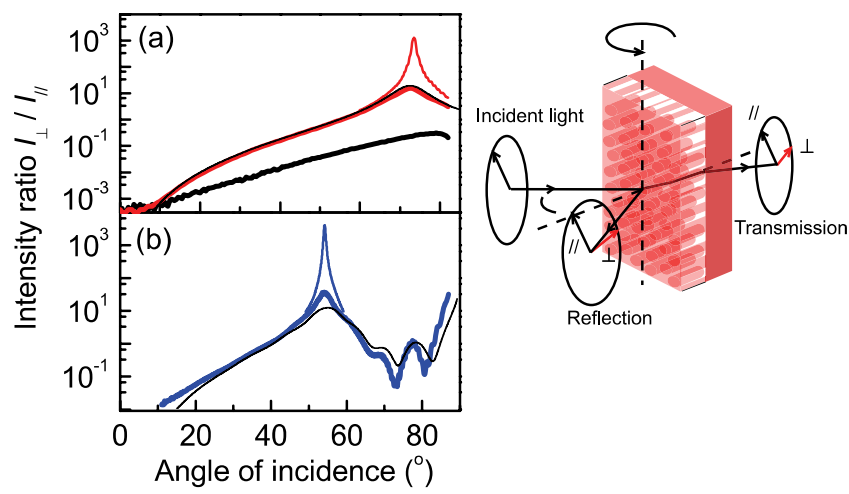

Figure 5. (a) Transmission intensity ratio $I_{\perp} / I_{/ /}$as a function of the angle of incidence for sample $\mathrm{B}$ (red line) and for a bare GaP substrate (thick black line). (Thin black line) denotes fit using a transfer-matrix model of a birefringent layer. (b) Ratio $I_{\perp} / I_{/ /}$for the specular reflected light for sample B (blue line) and model fit (thin black line). Thin colored lines in (a) and (b) are measurements after optimization of the input polarization minimizing $\mathbb{I}_{/ /}$(see text). Sketch shows experimental configuration.

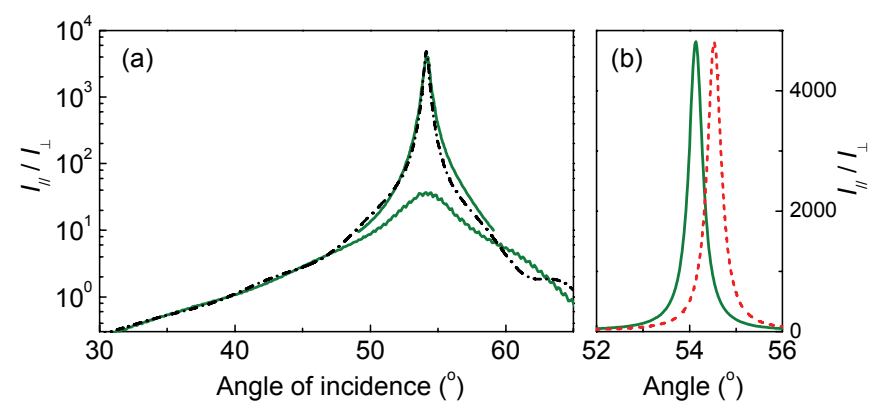

Figure 6. (a) Experimental (green lines) and calculated (dashdotted line, black) reflection contrast of sample B. (b) Calculated values around maximum at $54.1^{\circ}$ (linear scale) for a birefringence $\Delta \mathrm{n}=0.047$ (green) and for $\Delta \mathrm{n}=0.046$ (dashed line, red).

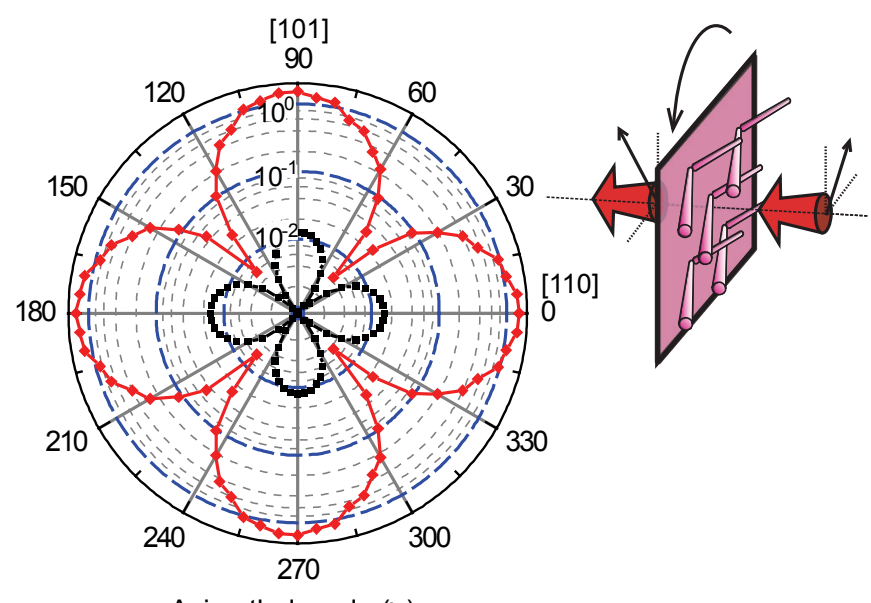

Azimuthal angle (o )

Figure 7. Transmitted intensity ratio $I_{\perp} / I_{/ /}$at a wavelength of $690 \mathrm{~nm}$, as a function of the azimuthal orientation of the birefringent nanowire layer, for $\mathrm{GaP}$ nanowires $\mathrm{C}$ (black squares) and $\mathrm{F}$ (red diamonds) grown on (100) GaP (see Fig. 2a and b). Sketch shows experimental configuration.
Received: ((will be filled in by the editorial staff))

Revised: ((will be filled in by the editorial staff)) Published online: ((will be filled in by the editorial staff))

[1] N. Engheta, R. W. Ziolkowski, Electromagnetic metamaterials: physics and engineering explorations; 1st edition, Wiley, New York 2006.

[2] M. Born, E. Wolf, Principles of Optics, 6th ed., Cambridge University Press, Cambridge 1997, 665.

[3] W. A. De Heer, W. S. Bacsa, A. Chatelain, T. Gerfin, R. HumphreyBaker, L. Forro, D. Ugarte, Science 1995, 268, 845.

[4] N. Künzner, D. Kovalev, J. Diener, E. Gross, V. Y. Timoshenko, G. Polisski, F. Koch, M. Fujii, Opt. Lett. 2001, 26, 1265.

[5] E. Gross, D. Kovalev, N. Künzner, V. Y. Timoschenko, J. Diener, F. J. Koch, J. Appl. Phys. 2001, 90, 3529.

[6] B. O, R. Liu, Y. Y. Li, M. J. Sailor, and Y. Fainman, IEEE Photon. Technol. Lett. 2003, 15, 834 .

[7] M. F. Weber, C. A. Stover, L. R. Gilbert, T. J. Nevitt, A. J. Oudekirk, Science 2000, 287, 2451.

[8] F. Genereux, S. W. Leonard, H. M. Van Driel, A. Birner, U. Gösele, Phys. Rev. B 2001, 63, 161101.

[9] H. Kosaka, T. Kawashima, A. Tomita, M. Notomi, T. Tamamura, T. Sato, S. Kawakami, Phys. Rev. B 1998, 58, R10096.

[10] F. Xu, R.-C. Tyan, P.-C. Sun, Y. Fainman, C.-C. Cheng, A. Scherer, Opt. Lett. 1995, 20, 2457.

[11] E. Peeters, J. Lub, J. A. M. Steenbakkers, and D. J. Broer, Adv. Mat. 2006, 18, 2412.

[12] Kotlyar, M.V.; Bolla, L.; Midrio, M.; O'Faolain, L.; Krauss, T.F. Opt. Express 2005, 13, 5040.

[13] V. S.-Y. Lin, K. Motesharei, K. S. Dancil, M. J. Sailor, M. R. Ghadiri, Science 1997, 278, 840 .

[14] S. E. Létant and M. J. Sailor, Adv. Mat. 2001, 13, 335.

[15] J.-Q. Xi, M. F. Schubert, J. K. Kim, E. F. Schubert, M. Chen, S.-Y. Lin, W. Liu and J. A. Smart, Nature Phot. 2007, 1, 176.

[16] Y. Li, F. Qian, J. Xiang, and C.M. Lieber, Mater. Today 2006, 9, 18; P. J. Pauzauskie and P. Yang, ibid., 36.

[17] J. Wang, M. K. Gudiksen, X. Duan, Y. Cui, C. M. Lieber, Science 2001, $293,1455$.

[18] O. L. Muskens, M. T. Borgström, E. P. A. M. Bakkers, and J. Gómez Rivas, Appl. Phys. Lett. 2006, 89, 233117.

[19] T. Mårtensson, C .P. T. Svensson, B. A. Wacaser, M. W. Larsson, W. Seifert, K. Deppert, A. Gustafsson, L. R. Wallenberg, L. Samuelson, Nano Lett. 2004, 4, 1987.

[20] E. P. A. M. Bakkers, J. A. Van Dam, S. De Franceschi, L. P. Kouwenhoven, M. Kaiser, M. Verheijen, H. Wondergem, P. Van der Sluis, Nature Mat. 2004, 3, 769.

[21] A. I. Hochbaum, R. He, R. Fan, P. Yang, Nano. Lett. 2005, 5, 457.

[22] M. P. Stewart, J. M. Buriak, Adv. Mat. 2000, 12, 859

[23] H. D. Tong, H. V. Jansen, V. J. Gadgil, C. G. Bostan, E. Berenschot, C. J. M. van Rijn, and M. Elwenspoek, Nano Letters 2004, 4, 283.

[24] M. Law, L. E. Greene, J. C. Johnson, R. Saykally, and P. Yang, Nature Mat. 2005, 4, 455.

[25] B. Tian, X. Zheng, T. J. Kempa, Y. Fang, N. Yu, G. Yu, J. Huang, and C. M. Lieber, Nature 2007, 449, 885 .

[26] I. Doron-Mor, Z. Barkay, N. Filip-Granit, A. Vaskevich, and I. Rubinstein, Chem. Mater. 2004, 16, 3476.

[27] Wagner, R. S.; Ellis, W. C. Appl. Phys. Lett. 1964, 4, 89.

[28] E.I. Givargizov, J. Cryst. Growth 1975, 31, 20.

[29] M. A. Verheijen, G. Immink, T. De Smet, M. T. Borgström, E. P. A. M. Bakkers, JACS 2006, 128, 1353.

[30] E. D. Palik, Handbook of optical constants of solids, 1st edition, Academic Press, Orlando 1985, 445.

[31] O. L. Muskens, J. Treffers, M. T. Borgström, E. P. A. M. Bakkers, and J. Gómez Rivas, Opt. Lett. 2007, 32, 2097.

[32] C. F. Bohren and D. R. Huffman, Absorption and scattering of light by small particles, 1st edition, Wiley-VCH, Weinheim 1998, 141.

[33] J. H. Burnett, Z. H. Levine, E. L. Shirley, Phys. Rev. B 2001, 64, 241102 
Layout 1:

\section{FULL PAPER}

Aligned semiconductor nanowires represent a novel class of photonic metamaterials. We fabricated dense nanowire layers with controlled alignment, showing giant optical birefringence in the visible spectrum. These artificial materials hold promise for applications in optical sensing and photonics.

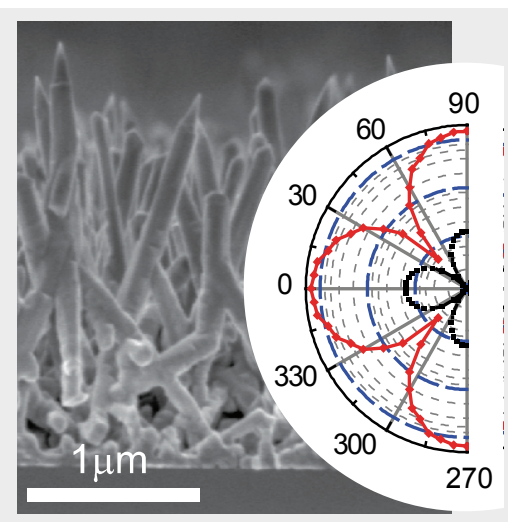

Nanowire photonic metamaterials with controlled density and alignment show giant optical birefringence

O. L. Muskens, S. L. Diedenhofen, M. H. M. van Weert, M. T. Borgström, E. P. A. M. Bakkers, and J. Gómez Rivas ${ }^{*}$ Page No. - Page No.

Epitaxial growth of aligned semiconductor nanowire metamaterials for photonic applications 